\title{
Aspirine en prévention primaire
}

\author{
Jean-Michel Juliard \\ Hôpital Bichat, Service de cardiologie, Paris, France \\ jean-michel.juliard@aphp.fr
}

$\mathrm{n}$ prévention secondaire après un accident ischémique vasculaire, le bénéfice clinique de l'aspirine est fondé sur des preuves scientifiques solides contrebalançant efficacement le risque hémorragique. Par contre, le bénéfice de l'aspirine en prévention primaire fait encore débat malgré de nombreuses études randomisées ayant inclus plusieurs dizaines de milliers de patients. Dans une méta-analyse de 118445 patients issus de 11 essais thérapeutiques, l'aspirine, en prévention primaire, permet une réduction relative du risque d'infarctus du myocarde (IDM) de $22 \%$, de décès de $6 \%$ avec un prix à payer : l'augmentation du risque hémorragique digestif de $59 \%$ et d'accident vasculaire cérébral (AVC) hémorragique de $33 \%[1,2]$.

En prévention primaire ne faut-il pas restreindre l'indication de l'aspirine aux patients « à risque », ayant un ou plusieurs facteurs de risque ? Deux études récentes ont ciblé cette indication, l'une faite chez le diabétique (étude ASCEND) et l'autre chez les patients à risque cardiovasculaire modéré (étude ARRIVE).

L'étude ASCEND est une étude randomisée effectuée chez 15480 patients (> 40 ans) diabétiques (94\% de diabète de type II, sans antécédent d'événement cardiovasculaire), comparant $100 \mathrm{mg} / \mathrm{j}$ d'aspirine contre placebo [3]. Le critère primaire d'efficacité était la survenue du premier événement : IDM, AVC, décès d'origine vasculaire. Le critère primaire de sécurité était la survenue du premier accident hémorragique grave : hémorragie intracrânienne, hémorragie oculaire, digestive ou toute autre hémorragie grave. Le critère secondaire comportait le risque de cancer digestif. Sur un suivi moyen de 7,4 années, le taux d'événements était plus bas dans le groupe aspirine $(8,5 \%$ vs $9,6 \%$, $p=0,01)$ au prix d'un risque hémorragique plus élevé $(4,1 \%$ vs $3,2 \%$, $p=0,003)$, essentiellement au niveau digestif. Les taux de cancers digestifs étaient identiques entre les deux groupes $(2,0 \%)$. Concernant la mortalité vasculaire, il n'y avait pas de différence non plus entre aspirine et placebo $(2,5 \%$ vs $2,8 \%)$.

Le schéma de l'étude ARRIVE était comparable à celui de l'étude ASCEND, comparant aspirine $100 \mathrm{mg} / \mathrm{j}$ au placebo avec les mêmes critères d'efficacité et de sécurité [4]. Par contre, les patients diabétiques étaient exclus ainsi que les patients à haut risque hémorragique digestif. Les patients inclus (12 546, hommes > 55 ans, femmes > 60 ans) étaient à risque cardiovasculaire modéré avec un score de Framingham à haut risque de maladie coronaire estimé à $14 \%$ à 10 ans dans chaque groupe, et $28 \%$ étaient fumeurs, $65 \%$ hypertendus et $45 \%$ avaient un LDL cholestérol élevé. Après un suivi moyen de cinq ans, les taux d'événements cardiovasculaires étaient identiques entre les deux groupes $(4,29 \%$ vs $4,48 \%$, $p=0,6038)$ mais avec un risque accru d'hémorragies digestives $(0,97 \%$ vs $0,46 \%, p=0,0007)$. Les risques de décès étaient identiques entre les deux groupes : 160 dans le groupe aspirine contre 161 dans le groupe placebo.
Les résultats de ces deux études ne permettent pas de recommander l'utilisation de l'aspirine en prévention primaire, que ce soit chez le sujet diabétique ou le patient à risque cardiovasculaire modéré. Le bénéfice clinique le plus substantiel est la réduction des IDM mais sans impact significatif sur la mortalité. Il n'y a pas de réduction non plus du risque d'AVC. Le taux d'événement observé était largement inférieur à celui estimé à long terme, comme dans l'étude ARRIVE où le score de Framingham utilisé n'est plus probablement aussi pertinent en 2018 qu'à l'époque où il a été construit. Le traitement plus intensif des facteurs de risque, comme l'hypertension artérielle ou les dyslipidémies, contribue probablement à réduire conjointement à l'aspirine le risque cardiovasculaire à long terme.

Il faut donc rester sur les recommandations 2016 de la Société européenne de cardiologie (ESC) sur la prévention cardiovasculaire : l'aspirine n'est pas justifiée en prévention primaire, en l'absence d'antécédent ischémique, compte tenu du risque hémorragique [5]. D'autres sousgroupes de patients, à plus haut risque cardiovasculaire (qu'il reste encore à définir), justifieraient-ils cette prévention primaire ? Des études ultérieures sont nécessaires pour le prouver.

Liens d'intérêts : l'auteur déclare ne pas avoir de lien d'intérêt.

\section{Références}

1. Guirguis-Blake JM, Evans CV, Senger CA, O'Connor EA, Whitlock EP. Aspirin for the primary prevention of cardiovascular events ; 
a sytematic evidence review for the US preventive services task force. Ann Intern Med 2016 ; 164 : 804-13.

2. Whitlock EP, Burda BU, Williams SB, Guirguis-Blake JM, Evans CV. Bleeding risks with aspirin use for primary prevention in adults : a systematic review for the US preventive services task force. Ann Intern Med $2016 ; 164: 826$.
3. The ASCEND Study Collaborative Group. Effects of aspirin for primary prevention in persons with diabetes mellitus. $N$ Engl J Med 2018 ; 379(16) : 1529-39.

4. Gaziano JM, Brotons C, Coppolecchia R, et al., the ARRIVE Executive Committee. Use of aspirin to reduce risk of initial vascular events in patients at moderate risk of cardiovascular disease (ARRIVE) : a randomised, double-blind, placebo-controlled trial. Lancet 2018 ; 392(10152) : 1036-46.

5. Piepoli MF, Hoes AW, Agewall S, et al. 2016 European guidelines on cardiovascular disease prevention in clinical practice : the sixth joint task force of the European Society of Cardiology and other societies on cardiovascular disease prevention in clinical practice. Eur Heart J 2016 ; 37 : 2315-81.

\title{
Les dix commandements des recommandations de la Société européenne de cardiologie sur le traitement de l'hypertension artérielle de l'adulte
}

\author{
Jean-Michel Juliard \\ Hôpital Bichat, Service de cardiologie, Paris, France \\ jean-michel.juliard@aphp.fr
}

es nouvelles recommandations

2018 de la Société européenne

de cardiologie (ESC) sur la prise en charge de l'hypertension artérielle (HTA) de l'adulte viennent d'être publiées. Mais avec plus de 80 pages et plus de 600 références bibliographiques, il n'est pas toujours facile d'adapter ces recommandations à notre pratique quotidienne. En cela, il faut remercier deux auteurs (Bryan Williams et Giuseppe Mancia) d'avoir résumé en dix « commandements » toutes ces considérations $[1,2]$.

\section{Définir l'hypertension artérielle de l'adulte}

L'HTA est définie en cas de pression artérielle systolique $\geq 140 \mathrm{et} / \mathrm{ou}$ pression diastolique $\geq 90 \mathrm{mmHg}$ au cabinet, correspondant à une pression artérielle moyenne au cours d'un enregistrement Holter $\geq 130 / 80$ $\mathrm{mmHg}$ ou une auto-mesure $\geq 135 / 85$ $\mathrm{mmHg}$.

\section{Dépistage et diagnostic}

La mesure de la pression artérielle devrait être faite au moins une fois tous les cinq ans chez un adulte et plus fréquemment en cas de pression artérielle élevée. Quand une HTA est suspectée, les mesures doivent être répétées au cours de plusieurs consultations espacées dans le temps et confirmées par un enregistrement Holter et des auto-mesures.

Quand considérer la mise en route d'un traitement ?

En cas d'HTA grade 1 (mesurée au cabinet 140-159/90-99 mmHg) chez un adulte (jusqu'à 80 ans), si cette HTA persiste après une période d'intervention hygiéno-diététique. En cas d'HTA grade 1 avec risque cardiovasculaire élevé ou HTA grade $2(\geq 160 / 100 \mathrm{mmHg})$, un traitement antihypertenseur doit être institué parallèlement aux mesures hygiénodiététiques.

\section{Considérations spéciales : patients âgés}

Au-delà de 80 ans, les patients « vierges » de tout traitement et présentant une pression artérielle systolique $\geq 160 \mathrm{mmHg}$ doivent être considérés individuellement pour la mise en route d'un traitement. L'âge ne doit jamais être à lui seul un argument pour ne pas mettre en route un traitement ou arrêter un traitement prescrit préalablement.

\section{Objectifs thérapeutiques}

La cible thérapeutique doit être une pression artérielle systolique $<140$ $\mathrm{mmHg}$ dans tous les cas, même chez le sujet âgé, voire $<130 \mathrm{mmHg}$ en cas de bonne tolérance du traitement. Une cible thérapeutique $<130$ $\mathrm{mmHg}$ peut être justifiée chez le sujet $<65$ ans mais pas chez les sujets plus âgés. Des chiffres < $120 \mathrm{mmHg}$ ne sont pas recommandés en raison d'un mauvais rapport bénéfice/ risque. Dans tous les cas, l'objectif pour la pression artérielle diastolique est $<80 \mathrm{mmHg}$. Chez le patient diabétique, les mêmes objectifs sont recommandés.

\section{Mesures hygiéno-diététiques}

Ces mesures sont indispensables car elles peuvent contribuer à retarder la mise en route d'un traitement au long cours et s'imposent également avec un traitement antihypertenseur à atteindre l'objectif thérapeutique. Réduire sa consommation de sel et d'alcool, manger « sainement », 
c'est-à-dire une alimentation variée, conjointement à une activité physique régulière, contrôler son poids et l'arrêt du tabac sont autant de mesures bénéfiques à long terme audelà même du simple traitement de l'hypertension artérielle.

\section{Mise en route du traitement}

Fait nouveau dans ces dernières recommandations est celui d'instaurer d'emblée une bithérapie, compte tenu du fait que dans la plupart des cas l'objectif thérapeutique n'est pas atteint avec une monothérapie. Exception faite pour des sujets à faible niveau tensionnel initial ou patients âgés ou « fragiles » chez qui une monothérapie permet d'atteindre d'emblée la cible thérapeutique ? Mais en pratique, cela ne devrait concerner qu'un faible nombre de patients.

\section{Monoprise}

Le succès d'une thérapeutique au long cours instituée dans un cadre préventif à long terme est une prise par jour dans un seul comprimé permettant d'avoir une compliance optimale. Que ce soit en bi- ou trithérapie, le choix préférentiel est d'avoir les principes actifs dans un seul comprimé.

\section{Algorithme de traitement}

La bithérapie doit associer un vasodilatateur (inhibiteur de l'enzyme conversion ou des récepteurs à l'aldostérone) à un diurétique type thiazidique ou un inhibiteur calcique. En cas de trithérapie, ces trois principes actifs peuvent être associés dans la même présentation galénique. L'utilisation des bêtabloquants doit être restreinte en cas d'insuffisance coronaire, post-infarctus ou altération de la fonction ventriculaire gauche.

\section{Prise en charge du risque cardiovasculaire global chez l'hypertendu}

Au-delà du contrôle tensionnel, l'hypertendu a le plus souvent d'autres facteurs de risque à contrôler. L'utilisation conjointe de statines en cas d'hyperlipidémie, même modérée, s'est avérée bénéfique chez l'hypertendu. Par contre, l'utilisation de l'aspirine en prévention primaire n'est pas recommandée.

Ce résumé ne peut pas prendre en compte tous les cas de figure rencontrés dans la pratique quotidienne mais a le mérite de schématiser la prise en charge de la majorité de nos hypertendus. La notion d'HTA « résistante » est probablement le point le plus délicat, nécessitant de vérifier que le patient prend bien son traitement, qu'il est donné à des doses thérapeutiques et que son évaluation est correcte. C'est dans ce cas seulement que l'on peut ajouter de petites doses de spironolactone et aboutir alors à une quadrithérapie. Le recours à des stratégies non médicamenteuses, type dénervation rénale, n'est pas recommandé en routine, compte tenu de la pauvreté des données scientifiques. Une prise en charge dans un milieu spécialisé est alors nécessaire.

Liens d'intérêts : l'auteur déclare ne pas avoir de lien d'intérêt.

\section{Références}

1. Williams B, Mancia G, Spiering W, et al. 2018 ESC/ESH guidelines for the management of arterial hypertension. The Task Force for the management of arterial hypertension of the European Society of Cardiology (ESC) and the European Society of Hypertension (ESH). Eur Heart J 2018 ; 39 : 3021-104. 2. Williams B, Mancia G. Ten commandments of the 2018 ESC/ESH HTN guidelines on hypertension in adults. Eur Heart $J 2018$; 39 : 3007-19.

\section{Traitement percutané de l'insuffisance mitrale secondaire}

\section{Jean-Michel Juliard}

Hôpital Bichat, Service de cardiologie, Paris, France

jean-michel.juliard@aphp.fr

u cours de l'insuffisance cardiaque, le ventricule gauche se dilate, ultérieurement l'anneau mitral, distendant ensuite les cordages de la valve mitrale, et ainsi le bord libre de ces valves ne s'affronte plus, et se développe alors une insuffisance mitrale (IM) dite secondaire ou fonctionnelle car les feuillets valvulaires sont anatomiquement normaux. Cette IM secondaire contribue à aggraver la congestion pulmonaire ; réhospitalisations, altération de la qualité de vie et réduction de la survie en sont les conséquences immédiates et à long terme. Malgré les thérapeutiques médicales (diurétiques, vasodilatateurs, bêtabloquants) associées à la resynchronisation, si possible, certains patients restent symptomatiques, NYHA classe II à IV. Le risque opératoire 


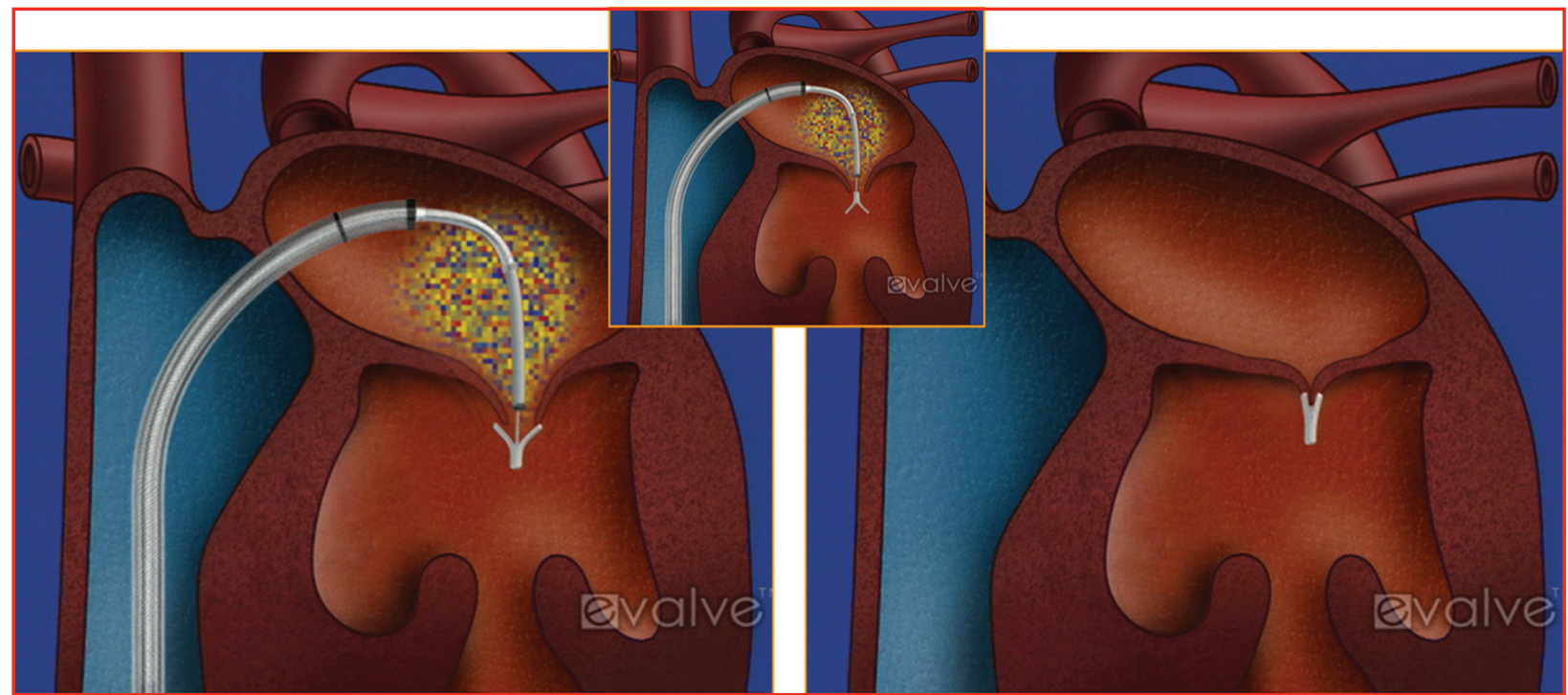

Figure 1. Le système MitraClip ${ }^{\circledR}$ consiste à implanter, par voie transeptale, un clip sur les deux bords libres des valves mitrales créant ainsi un double orifice ayant pour objectif de réduire la fuite mitrale (en ayant soin de ne pas créer une sténose mitrale !). Cette intervention percutanée reproduit la méthode développée par le chirurgien cardiaque Alfiéri qui effectuait cette intervention à coeur ouvert, avec des résultats qui étaient très encourageants (technique d'Alfiéri).

de la correction isolée de l'IM secondaire est lié à l'altération de la fonction ventriculaire gauche. La mortalité augmente linéairement avec l'abaissement de la fraction d'éjection ventriculaire gauche (FEVG). En comparaison à la technique percutanée utilisant le MitraClip ${ }^{\circledR}$ (figure 1) dans l'étude EVEREST, il n'y a pas de différence significative avec la chirurgie au cours de l'IM secondaire, alors que le MitraClip ${ }^{\circledR}$ est plus favorable que la chirurgie au cours des IM organiques [1].

En France, contrairement à l'Allemagne, le remboursement du MitraClip ${ }^{\circledR}$ est restreint aux IM organiques. Deux études récentes, MITRA-FR et COAPT, ont comparé le MitraClip ${ }^{\circledR}$ au cours des IM secondaires au traitement médical seul chez des patients symptomatiques. Les patients du groupe interventionnel recevaient également une thérapeutique médicale optimale (médicaments \pm resynchronisation) $[2,3]$.

L'étude MITRA-FR, récemment présentée au dernier congrès européen de cardiologie à Munich, est une étude française financée par le ministère de la Santé dans le cadre d'un programme hospitalier de recherche clinique (PHRC). Les patients inclus devaient avoir une FEVG entre 15 et $40 \%$ avec une IM significative définie par une surface régurgitante $>20 \mathrm{~mm}^{2}$ et un volume régurgitant $>30 \mathrm{ml} /$ battement. Les patients ont été randomisés entre intervention percutanée avec le MitraClip ${ }^{\circledR}(\mathrm{n}=152)$ et traitement médical $(n=152)$. Le critère de jugement principal associait décès de toutes causes et réhospitalisations pour insuffisance cardiaque à un an. Il y a eu six échecs d'implantation dans le groupe interventionnel. Au final, 43 patients ont été exclus dans le groupe interventionnel et seuls 109 d'entre eux ont eu l'intervention. Il n'y a pas eu de différence entre les deux bras : 54,6\% dans le groupe interventionnel contre $51,3 \%$ dans le groupe médical $(p=0,53)$. À un an, les taux de mortalité étaient également comparables entre les deux groupes
(24,3\% vs $22,4 \%)$. En analyse de sous-groupes (14 au total), il n'y avait aucune interaction, les résultats étaient homogènes.

L'étude COAPT, menée aux ÉtatsUnis, présentée au dernier congrès Transcatheter Cardiovascular Therapeutics (TCT) à San Diego, a eu le même schéma : patients symptomatiques, classe NYHA > 2, FEVG entre 20 et $50 \%$, IM sévère grade III ou IV, traités de façon optimale sur le plan médical. Entre 2012 et 2017, 614 patients ont été inclus dans 78 centres et randomisés entre une stratégie interventionnelle $(\mathrm{n}=302)$ ou médicale seule $(n=312)$. Le critère primaire de jugement était le taux de réhospitalisations pour insuffisance cardiaque à deux ans. À deux ans, le bénéfice était clairement en faveur de la stratégie interventionnelle avec un taux de réhospitalisations de $35,8 \%$ vs $67,9 \%$ dans le groupe médical $(p<0,001)$. La mortalité était également réduite dans le groupe interventionnel $(29,1 \%$ vs $46,1 \%$, $p<0,001)$. En analyse de sous-groupe, 
il n'y avait pas d'interaction, notamment avec la fonction VG, ni avec la gravité de l'IM. Le bénéfice de la stratégie interventionnelle n'était pas retrouvé chez les femmes qui représentaient environ $40 \%$ de la cohorte de patients. Ces deux études ont donc, apparemment, des résultats contradictoires quant au bénéfice du MitraClip ${ }^{\circledR}$ vs le traitement médical en cas d'IM fonctionnelle sévère symptomatique chez des patients ayant une altération de la fonction $\mathrm{VG}$, avec une FEVG qui était en moyenne de $33 \%$ dans l'étude MITRA-FR et $31 \%$ dans l'étude COAPT. Le bénéfice observé est absolument incroyable dans l'étude COAPT, car le nombre de patients à traiter pour éviter une hospitalisation est de trois et pour prévenir un décès de six ! Telle est une vraie révolution thérapeutique ! Cela mérite réflexion car à un an les mortalités globales étaient comparables entre MITRA-FR (22\%) et COAPT (23\%). Si l'on regarde les mortalités à un an dans l'étude COAPT (le critère principal était à deux ans), elles n'étaient pas différentes entre le groupe interventionnel et médical $(19,1 \%$ vs $23,2 \%$, OR 0,81 , IC $95 \%=0,57-1,15$ ), et finalement assez proches des mortalités observées à un an dans l'étude MITRA-FR. Tout le bénéfice apparaîtrait entre un et deux ans ?

Une autre étude européenne est actuellement en cours, étude RESHAPE-HF, et devrait inclure environ 400 patients, car de tels résultats discordants méritent encore de connaître chez ces patients ce qui revient à la pathologie mitrale et à la pathologie myocardique.

Liens d'intérêts : l'auteur déclare ne pas avoir de lien d'intérêt.

\section{Références}

1. Feldman T, Foster E, Glower DD, et al. Percutaneous repair or surgery for mitral regurgitation. N Engl J Med 2011 ; 364 : 1395-406.

2. Obadia JF, Messika-Zeitoun D, Leurent $\mathrm{G}$, et al., for the MITRA-FR investigators. Percutaneous repair or medical treatment for secondary mitral regurgitation. $N$ Engl J Med 2018. Doi : 10.1056/NEJMoa1805374. [Epub ahead of print].

3. Stone GW, Lindenfeld JA, Abraham WT, et $a l$., for the COAPT investigators. Transcatheter mitral-valve repair in patients with heart failure. N Engl J Med 2018. Doi : 10.156/ NEJMoa1806640. [Epub ahead of print]. 